\title{
PANORAMA DE LA GESTIÓN DE LA CALIDAD ACADÉMICA UNIVERSITARIA
}

Albert Ypma*

En este artículo se analiza de manera práctica el papel de los funcionarios a cargo de la gestión académica universitaria. El artículo comprende tres aspectos. Primero: una mirada estratégica dónde se discuta sobre la Sociedad del Conocimiento, el perfil de los egresados en el mercado laboral y la política europea sobre la educación superior. Segundo: el panorama de la calidad con el modelo de gestión de la European Foundation of Quality Management y el ciclo de Deming: planificar, hacer, medir y mejorar. Y finalmente el papel estimulante de la gestión académica en desarrollar un modelopedagógico institucional para la aplicaión en los planes de estudio y las formas de enseñanza y aprendizaje y en desarrollar un ambiente de calidad académica. El mismo se basa en los resultados de los intercambios con compañeras y compañeros de la Universidad Nacional de Costa Rica durante los talleres del proyecto MHO Desarrollo de la Calidad Institucional, asi como en otras experiencias holandesas.
Practical analysis of the role played by the staff in charge of the university's academic management. Three aspects are covered. First, a strategic view to discuss the Knowledge Society, the profile required of alumni in the work market and the European policy concerning Higher Education. Second, an outlook on quality, based on the management model from the European Foundation of Quality Management and the Deming cycle: planning, doing, measuring and improving. And finally, the stimulating role of academic management in developing an institutional, pedagogical model to be applied in study programs in the different teaching and learning methods, and in developing an atmosphere of academic quality. The model is the result of exchanges among coworkers of Universidad Nacional in the workshops organized by the MHO project Developing Institutional Quality, and also through other Dutch experiences.

* Exdirector académico de la Universidad Politécnica Leiden, Holanda (1990-2002). Asesor en el proyecto MHO Desarrollo de la Calidad Institucional (1995-2004). Master of Arts en Ciencias de Lingüística y Letras, Universidad de Groningen, Holanda, y ha estudiado postgrados en Ciencias Educativas y Didácticas, y en Gestión de la Calidad para organizaciones sin fines de lucro. Ahora, en Honduras, es asesor en educación y representante de la CHN Universidad Politécnica, Leeuwarden, Holanda. 


\section{La mirada estratégica}

¿Por qué empezamos con el enfoque estratégico? Un motivo es el desafío para las universidades de formular una respuesta a la tendencia global que la sociedad, o las diferentes sociedades con sus ritmos diferentes, cambia fundamentalmente hacía una sociedad basada en el conocimiento. El volumen de conocimientos en el mundo se duplica cada cuatro o cinco años. En materias como informática, telecomunicaciones y biotecnología dicho avance se materializa en tan solo dos años ${ }^{1}$. Algunos expertos salvadoreños dicen:

Para ser competitivo en el mundo de hoy, un país debe aprender no sólo cómo producir y vender mejor, sino también - y de manera urgente -: cómo manejar su riqueza de conocimiento, cómo generar nuevos conocimientos, cómo traducir el conocimiento en información útil para los objetivos del desarrollo y de la competitividad, y de cómo captar información externa y aplicarla localmente en provecho de sus fines ${ }^{2}$.

Dado el fuerte papel tradicional de las universidades en la producción, la enseñanza y el aprendizaje del conocimiento, ellas deberían tener un rol importantísimo en transformar un país. Pero las demandas de la Sociedad del Conocimiento no son realmente claras. Entonces una universidad, para dar la respuesta adecuada, debe analizar profundamente el entorno para desarrollar su estrategia. Otro motivo para la mirada estratégica es la competitividad creciente entre universidades públicas y privadas. Una universidad debe definir su posición deseable. Ser mejor que otras universidades, o ser diferente de otras universidades, u ojalá ser mejor y diferente ${ }^{3}$.

El marco de referencia sobre el desarrollo estratégico, derivado de la Universidad Libre de Bruselas, sirve para conocer la contribución necesaria de una gestión académica, el cual no es solamente ser un ente administrativo más. En este marco analítico se divide el terreno estratégico - entorno, políticas y cultura- en tres ámbitos. El macro: la institución como parte y aliada de los procesos del desarrollo nacional, regional y global; el ámbito meso: la universidad como un conjunto unitario; y el ámbito micro: la unidad académica, la carrera o las escuelas universitarias.

\footnotetext{
'Sociedad del Conocimiento', www.sociedaddelconocimiento.net

'Estrategia para la Creación de una Sociedad de Aprendizaje'. El Salvador, 1999.

'Organizational adaptation in higher education', in: 'From the Eye of the Storm, Higher Education's Changing Institution'. CHEPS, 1999.
} 


\begin{tabular}{|c|c|c|c|}
\hline & $\begin{array}{c}\text { Macro } \\
\text { Nacional + }\end{array}$ & $\begin{array}{c}\text { Meso } \\
\text { Institución }\end{array}$ & $\begin{array}{l}\text { Micro } \\
\text { Carrera }\end{array}$ \\
\hline Enterno & $\begin{array}{l}\text { Tendencias en la } \\
\text { sociedad y el } \\
\text { mercado laboral }\end{array}$ & $\begin{array}{l}\text { Posición de la } \\
\text { universidad como } \\
\text { institución de } \\
\text { educación superior }\end{array}$ & $\begin{array}{c}\text { Características de los } \\
\text { estudiantes }\end{array}$ \\
\hline Políticas & $\begin{array}{l}\text { Políticas del gobiemo } \\
\text { sobre la educación } \\
\text { superior }\end{array}$ & $\begin{array}{c}\text { Misión y visión de la } \\
\text { universidad }\end{array}$ & Planes de estudio \\
\hline Cultura & $\begin{array}{l}\text { Tendencias en la } \\
\text { educación y la } \\
\text { acreditación }\end{array}$ & $\begin{array}{l}\text { Ambiente de la } \\
\text { calidad }\end{array}$ & $\begin{array}{c}\text { Formas de enseñanza } \\
\text { y aprendizaje }\end{array}$ \\
\hline
\end{tabular}

Vamos a mirar a la universidad desde afuera, desde el ámbito macro. Seleccionamos una tendencia acerca del mercado laboral y de la política del gobierno sobre la educación superior.

¿Cuál perfil de los egresados quiere el mercado laboral? Las empresas y organizaciones comprenden más que nunca que los egresados no solo deben tener más conocimientos sino otros conocimientos: deben tener la habilidad de aprender rápidamente nuevos conocimientos, “aprender a aprender". El perfil incluye también otras habilidades, dirigidas a la aplicación del conocimiento. Por ejemplo, un auditor debe tener una Licenciatura en Contaduría Pública, y también habilidades técnicas como ser analítico y capaz de usar herramientas modernas de gestión (como el Balanced Scorecard), pensar estratégicamente y manejar la tecnología computacional. Son importantes las características personales como ser proactivo, ser emprendedor, ser comunicativo y mantener buenas relaciones interpersonales, estar orientado al desarrollo profesional propio, y poseer dominio -al lado del español- del idioma inglés ${ }^{4}$. Es interesante comparar este perfil con la percepción de estudiantes distinguidos ${ }^{5}$ de la Universidad Nacional, durante un taller donde se discutió acerca de las características de los futuros estudiantes. Ellos señalan entre otras: la habilidad en el uso de la tecnología e idiomas; ser analítico y crítico; y hábil y creativo para encontrar soluciones ágiles; además capacidad de trabajar en equipo, con actitud investigativa y manejo de metodologías; preocupado por el ambiente y la naturaleza (Universidad Nacional, 2003).Hasta ahora faltan mecanismos para

Anuncio en El Heraido, Honauras, el 15 de febrero de 2004.

5 Los estudiantes distinguidos son los que la Universidad reconoce por el excelente desempeño académico. 
identificar las competencias de los profesionales para definir perfiles convenientes. Las generalizaciones sobre las demandas del mercado laboral y las ideas de estudiantes distinguidos exigen adaptaciones en el currículum actual que normalmente se basan en el desarrollo de contenidos o disciplinas tradicionales y en el desempeño de los profesores que, en muchos casos, aún prefieren la memorización a la comprensión.

La política de los gobiernos centroamericanos sobre la educación superior, que puede influir a la universidad como institución, es menos clara o aún ausente. ${ }^{6}$ Quizás por la tradición de la autonomía de las universidades, los ministros de la Educación limitan sus políticas al nivel de la educación básica y secundaria. Las universidades centroamericanas prefieren no contar con lineamientos de sus gobiernos nacionales y aún menos de instancias políticas regionales, como el Parlacen. En el marco de la competitividad existen planes oficiales para el desarrollo de los recursos humanos. La meta principal del Plan Puebla-Panamá para el sur de México, Guatemala, Honduras, El Salvador, Nicaragua, Costa Rica, Belice y Panamá, es "mejorar la competitividad de esos países y su inserción en el comercio del mundo, así aprovechar de forma más efectiva la enorme riqueza humana y ambiental de la región. Para ello se impulsan proyectos que conectan a todos los países de América Central y promueven el desarrollo humano de la población". ${ }^{7}$ Pero, sin una política universitaria mesoamericana, estas palabras no podrán ser traducidas en obras.

Al contrario, la Unión Europea y los ministros de Educación de 29 países europeos tienen desde la Declaración de Bologna (1999), una política muy clara sobre el papel de las universidades. Luego de llegar a la conclusión de que para competir económica y culturalmente con EE.UU., los ministros de educación de esos países decidieron que es mejor unir las fuerzas nacionales de la educación superior que dividirlas por las comprensibles razones de las diferencias en idiomas, formas históricas y niveles diferentes de calidad. Fue así como se siguió el camino de la armonización y comparabilidad en los sistemas de educación superior en Europa. Las universidades han aceptado el reto y trabajan en el desarrollo del nuevo sistema y su aplicación dentro de las instituciones. La meta es lograr en diez años -siguiendo pragmáticamente las reglas de juego angloamericanas- un sistema europeo con un bachillerato de 3 ó 4 años, una maestría de 1 ó 2 años y un doctorado de 3 a 4 años. El año académico será dividido en semestres, el European Credit Transfer System dicta pautas sobre el peso académico, sobre la puntuación del crédito y sobre la ejecución de proyectos europeos

\footnotetext{
6 Con respeto me refiero al trabajo del Consejo Superior de las Universidades Centroamericanas (CSUCA) para la política de una integración universitaria.

7 Tiempos del Mundo, 31 de julio de 2003.
} 
de investigación. Los objetivos específicos comprenden una mayor movilidad de estudiantes, docentes e investigadores y la en rada, en el mercado laboral, de profesionales mejor preparados. Un estudiante emprendedor puede elegir su ruta educativa en varias universidades del viejo continente que resultan interesantes para sus demandas intelectuales. Los ministros quieren estimular la competitividad entre las universidades con el desarrollo de un sistema europeo de acreditación capaz de diferenciar entre diversos niveles de calidad; por un lado las universidades realmente europeas y excelentes y por otro, las provinciales con una calidad mínima, pero suficientemente aceptable y en consecuencia, eliminar aquellas universidades o carreras sin calidad. Serían imaginables las ventajas de una política de los gobiernos mesoamericanos comparable con la de la Unión Europea y una gestión académica proactiva que pueda anticiparse a estas formas de internacionalización.

Si miramos hacia adentro, a los niveles meso y micro, vienen las preguntas estratégicas. ¿Qué es necesario para desarrollar una universidad que pueda jugar un papel en la Sociedad del Conocimiento y en el crecimiento de una competitividad regional sostenible? ¿Cuál debe ser la nueva misión universitaria para alcanzar una posición como universidad regional, atractiva con movilidad desde y hacia afuera de los mejores estudiantes, docentes e investigadores, y con una calidad realmente excelente?

\section{El panorama de la calidad}

En el proyecto MHO hemos usado como fuente de inspiración el modelo de la gestión de la calidad EFQM (European Foundation of Quality Management) y el ciclo de Deming: planificar, hacer, medir y mejorar (Figura 1), que es aplicable tanto al nivel de la institución, de una carrera o del desempeño individual de los funcionarios universitarios (vea el final del artículo). ${ }^{8}$

8 'Introducción a la excelencia' y 'Conceptos fundamentales de la excelencia', www.efgm.org y 'Método para mejorar la calidad de la educación superior basade en el modelo de la EFQM'. Club Gestión de Calidad, Madrid, 1999. Fuentes interesantes son también los libros, mencionados en la lista de referencias, de Paul James sobre 'Gestión de la Calidad Total' y V.M. Nava y A.R. Jiménez, 'ISO 9000:2000, Estrategias para implantar la norma de calidad para la mejora continua'. 


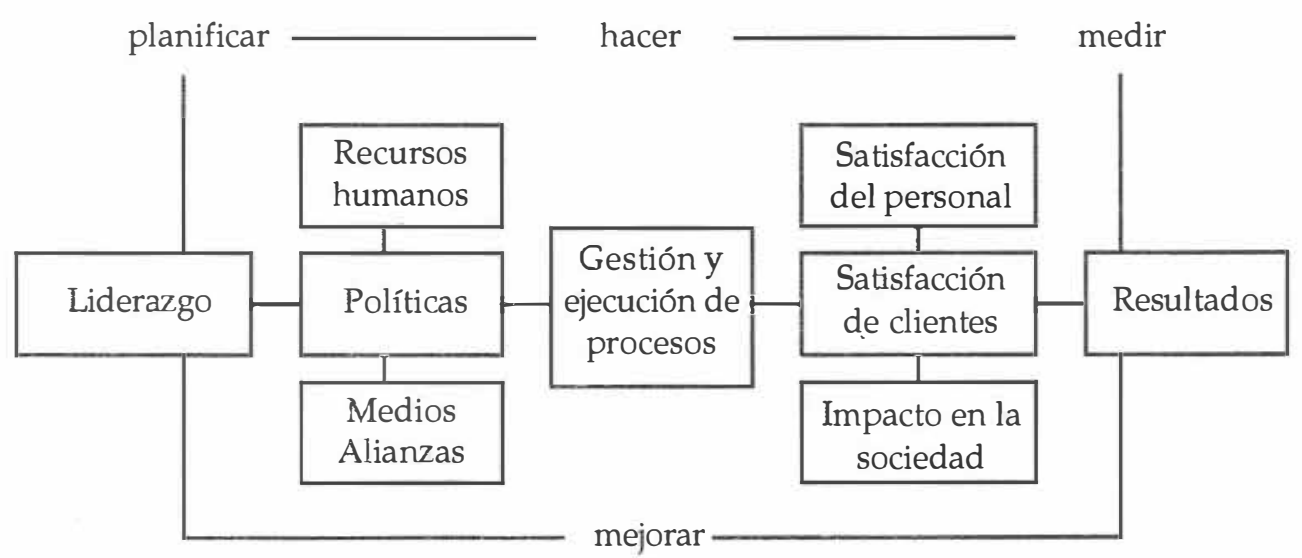

Figura 1. Modelo básico que combina el círculo de Deming con el Modelo de Gestión de la Calidad de la EFQM.

El modelo EFQM básico ilustra que el liderazgo proporciona dirección y contenido a la política y la estrategia, a la gestión de los recursos humanos y a la gestión de los medios y las alianzas. Estas son las condiciones organizativas que permiten llevar a la práctica, adecuadamente, la gestión de procesos entendidos como la enseñanza y aprendizaje, la extensión y la investigación. Los anteriores aspectos determinan el grado de satisfacción de los clientes, de la satisfacción del personal y del impacto en la sociedad. Estos aspectos, coherentes entre sí, conducen a los resultados institucionales deseados, entre los que se incluyen las mejoras en la calidad. Hay un desarrollo de cinco etapas de calidad o niveles de ambición o madurez que están interesantemente relacionados con los nueve criterios del sistema de la acreditación regional del CSUCA (SICEVAES). Estas cinco etapas de calidad son:

- La orientación a las actividades: cuando lo que predomina es "apagar incendios", la cultura informal de trabajo, las acciones individuales y las reacciones ad hoc.

- La orientación al proceso: la gestión de los proceso es la clave y la coordinación del proceso constituye el objetivo central (SICEVAES: transparencia).

- La orientación al sistema: la gestión abarca toda la organización: los servicios de apoyo, el trabajo en equipos responsables y se responde a una planificación a mediano plazo (se busca la coherencia, eficacia y eficiencia).

- La orientación a la cadena: amplía los vínculos entre la institución para "encadenarse" con los proveedores y los clientes. Hay interés por ser "embajador", crear impacto en la sociedad, y comparar el desempeño con respecto a otras entidades (benchmarking) para verificar la pertinencia y el impacto. 
- La orientación a la excelencia: hay un liderazgo visionario y una planifícación a largo plazo orientados al medio ambiente y la sostenibilidad. La institución es un punto de referencia para otros (universalidad, responsabilidad, equidad).

En los talleres MHO sobre la gestión académica se usa, al lado del modelo EFQM, dos principios como hilo conductor. La EFICACIA o 'hacer las cosas correctas' es la tarea especialmente de la planificación institucional y diaria. La EFICIENCIA o 'hacer correctamente las cosas' es prestar el servicio prometido con exactitud, seriedad, cortesía y en el momento oportuno; tener atención para la apariencia del personal, hacer uso adecuado de las instalaciones físicas, los equipos y los materiales y tener conciencia de los costos (minimizar los costos de los recursos necesarios para el logro de los objetivos).

\section{El papel de la gestión académica}

La gestión académica debe contribuir en general a la estrategia universitaria y a la gestión de la calidad, y específicamente debe estimular el desarrollo de un modelo pedagógico institucional y su aplicación en los planes de estudio y las formas de enseñanza y aprendizaje y en el desarrollo de un ambiente de calidad académica.

\section{Modelo pedagógico y su aplicación}

Muchas veces compiten dentro de una universidad o de una carrera diversos modelos pedagógicos, implícitos e individuales. El resultado son planes incoherentes de estudio, prácticas contradictorias de enseñanza y aprendizaje, y una confusión para los clientes. Los estudiantes no reciben una orientación pedagógico-didáctica clara y para los empleadores, los egresados no se distinguen de los egresados de otras universidades. Un modelo pedagógico explícito e institucional -o un modelo con un tronco común y variantes para las ciencias naturales, las ciencias sociales y el arte- es necesario para dar rumbo a las actividades de la enseñanza y del aprendizaje. La misión y visión actualizada de la universidad, las tendencias externas y teorías de educación (por ejemplo: el constructivismo) deben estar vinculadas a planes de estudio y formas adaptadas de enseñanza y aprendizaje. Además, el modelo sirve como hilo conductor durante la rendición de cuentas, en el proceso de acreditación y causa una mejor impresión de la imagen pública de una universidad. Por esto un modelo pedagógico debe ser un documento breve, que funciona como una guía estimulante para los docentes, estudiantes y autoridades. Un resumen en algunas pal abras claves o slogan capta la atención del público general, de los padres de familia, de los 
La gestión académica superior tiene la función de asesorar a las facultades en su tarea de formular, junto con

representantes del mercado laboral, de la sociedad y de los estudiantes interesados, las respuestas a las preguntas sobre el perfil de los profesionales ¿cuáles son los contextos del profesional?, ¿cuáles son sus roles? y ¿Cuáles son las competencias "amplias" convenientes? estudiantes potenciales y de los empleadores. Son ejemplos el "Aprender haciendo" de la Escuela Agrícola Panamericana Zamorano de Honduras, o el "Pensar y hacer" de la Universidad Politécnica de Rotterdam, Holanda?.

La Universidad Nacional elabora en estos años su modelo pedagógico. El punto de partida es el Plan Global Institucional que dice que el nuevo modelo se orienta a formar a los estudiantes mediante procesos comunicativos horizontales, experiencias vivenciales que permiten el aprendizaje en hacer, ser, aprender y convivir, incorpora la tecnología como recurso pedagógico y de trabajo y cuya calidad se instrumentaliza mediante procesos de acreditación. El interés expresado por losestudiantesdistinguidos en el taller antes dicho es salir de las aulas y realizar práctica en la realidad. Hasta ahora la Universidad Nacional tiene muchas carreras con un cuḿculumbasado en disciplinas. Los profesores imparten conocimientos y desarrollan habilidades desde las disciplinas en cursos separados; después los estudiantes deben aplicar la teoría en un periodo de práctica.

Para estructurar la gestión académica de un modelo pedagógico con el énfasis en "aprender a aprender" en lugar de "aprender contenidos" usamos tres preguntas fundamentales:

¿Qué se aprende? El desarrollo de los perfiles por competencias y no por los conocimientos de las disciplinas. Una competencia es la capacidad de una - persona de usar efectivamente una combinación de conocimientos, habilidades, actitudes y características personales (entre otras: su compromiso) para desempeñarse adecuadamente conforme a los requisitos de un contexto específico o de una tarea profesional, académica o cultural ${ }^{10}$.

¿Cómo se aprende? El estudiante es activo y no pasivo. Hace su plan personal de desarrollo y busca su ruta individual y flexible en la oferta de contextos,

\footnotetext{
9 La educación superior holandesa conoce dos tipos de universidades: Universidades Académicas con programas de Scientific Bachelor y Master y Universidades Politécnicas ('hogescholen') con programas de Professional Bachelor y Master.

10 Definición de la Universidad Politécnica de La Haya.
} 
tareas y problemas que requieren la integración de teoría y práctica y en cursos de apoyo. El profesor es una guía y un facilitador reflexivo.

¿Dónde se aprende? El ambiente de aprendizaje trasciende el aula y la biblioteca; es también el lugar de trabajo, por ejemplo, con trayectos duales; una plataforma virtual con recursos asociados a la información y comunicación: CDROM, Internet, correo electrónico y medios de comunicación, etc. ${ }^{11}$. Incluye también el espacio de la Vida Estudiantil con la formación integral durante debates, actividades culturales, expresiones de arte y el intercambio internacional.

La gestión académica superior tiene la función de asesorar a las facultades en su tarea de formular, junto con representantes del mercado laboral, de la sociedad y de los estudiantes interesados, las respuestas a las preguntas sobre el perfil de los profesionales ¿cuáles son los contextos del profesional?, ¿cuáles son sus roles? y ¿Cuáles son las competencias "amplias" convenientes? Luego las facultades con el apoyo central pueden reformular sus planes de estudio y formas de enseñanza y aprendizaje, en donde los contextos, desde la práctica, generan y facilitan el desarrollo individual de las competencias ${ }^{12}$. El currículum, por ejemplo, del primer año de la carrera de Economía y Logística en Rotterdam comprende cuatros proyectos derivados de cuatros competencias amplias: el gerente como estratega, arquitecto, líder y especialista. Mediante la clases de apoyo y de reflexión profesional se ofrece la base de conocimiento: el pensamiento conceptual de la carrera ('Pensar'). En los proyectos los alumnos aprenden a solucionar los problemas de la práctica profesional real mediante el uso del simulador de la cadena de transporte ('Hacer'). Finalmente hay posibilidades flexibles para los estudiantes con deficiencias en matemáticas, lenguas o el uso de computadores y el desarrollo de competencias personales por elegir. El ambiente de aprendizaje de la universidad agrícola hondureña está dado por seis "zamoempresas" como Lácteos y Cámicos en dónde los alumnos aprenden, haciendo veinte horas por semana y desarrollan la capacidad y disciplina del trabajo (trabajo en equipo, una comunicación efectiva, entender y abordar procesos en forma integral, resolver problemas y tomar decisiones) y su responsabilidad socioambiental ${ }^{13}$.

Para una implementación exitosa de un nuevo modelo pedagógico y un cumículum, es indispensable la capacitación de profesores en didáctica universitaria,

11 Véase sobrela plataforma virtual: OscarPicardo Joao. "Pedagogía informacional: enseñar a aprender enla sociedad del conocimiento".

12 Véase: M.E. Quesada Solano, M.A. Cedeño Suárez, J.M. Zamora Calvo. 'El diseño curricular en los planes de estu dio: aspectos teóxicos y guí metodológica’. Editorial Universidad Nacional, 2001.

13 Usamos estos ejernplos más por la claridad de su visión que por la concreción inmediata. Será diferente en universidades como ìa Universidad Nacional con su carácter diferente y más complicado. 
que además debe ser obligatoria. Pensamos entre otros, en un conocimiento del proceso psicológico del aprendizaje, en el desarrollo de conocimientos y habilidades en el diseño de un curso, en métodos de enseñanza y aprendizaje, en medios de instrucción y técnicas de evaluación. En general, la satisfacción de los estudiantes y del docente mismo, crecen inmediatamente si el docente no solamente tiene una formación académica en competencias científicas, sino también una formación profesional en competencias pedagógico-didácticas ${ }^{14}$.

\section{Ambiente de calidad académica}

Afortunadamente, la Universidad Nacional tiene un ambiente de calidad académica reconocido durante las primeras acreditaciones. Pero, siempre es necesario el desarrollo de este ambiente y el aseguramiento de la calidad, para lo cual se propone la siguiente metodología de gestión de la calidad ${ }^{15}$. El trabajo se debe establecer en dos ámbitos: el meso asegura la realización de la calidad institucional; en otras palabras, cumplir las metas estratégicas relacionadas con ese nivel de calidad y en el micro, donde se debe asegurar la calidad suficiente de las carreras para obtener la acreditación. El ciclo de Deming se usa simultáneamente conforme a los ciclos de la acreditación -cinco o seis años- y se vincula con el ciclo anual de planificación, de control y de rendición interna de cuentas:

Planificar: Contempla la formulación de indicadores concretos de la calidad basada en las metas institucionales y los indicadores externos de la acreditación.

Hacer: después una autoevalución, partiendo de la situación actual hacia las metas internas y externas, se ejecutan actividades de mejora, las cuales inician con la sensibilización.

Medir: se usan diversos instrumentos como entrevistas con estudiantes y egresados, evaluaciones del desempeño de los docentes, así como auditorías internas para medir los niveles de la calidad realmente alcanzados.

Mejorar: después de la medición, se formulan y ejecutan planes integrales de mejora, los cuales deben estar contemplados en los planes anuales presupuestales. Para costear los planes aprobados de mejora, debe existir una reserva presupuestaria institucional.

La Figura 2 provee un resumen del modelo de gestión de la calidad académica.

14 Véase: B. Matiru, A. Mwangi y R. Schlette. 'Teach your best. A Handbook for University Lecturers'.

15 Véase: 'Positionpaper 2003-2007'. Universidad Politécnica Rotterdam (texto en holandés). Su definición sencilla y efectiva de calidad es alcanzar las metas. 
5. Apoyar el trabajo para la acreditaci n: nivel de la calidad y ranking

\section{MBITO INTERNO}

3. Desarrollar

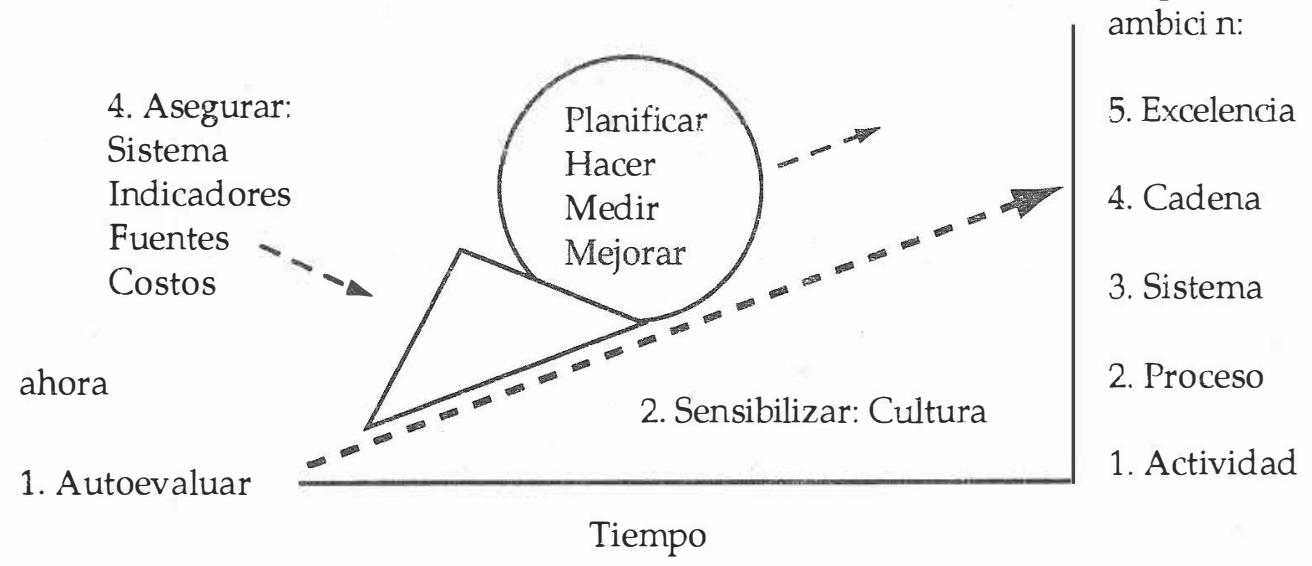

Figura 2. Adaptaci n del modelo de gesti n de la calidad a la gesti n de la oferta docente.

\section{Conclusión}

Hemos analizado un enfoque acerca de la gestión de la calidad en una institución académica, la cual debe estar dirigida a la ejecución efectiva y eficiente de los procesos claves de la universidad; particularmente a la elaboración e implementación de un modelo pedagógico institucional, de un currículum preferiblemente basado en competencias y de un ambiente de la calidad académica que garantice el logro de las metas institucionales y de la acreditación de las carreras. Bajo esta concepción de gestión de la calidad académica, se contribuye al desarrollo de los objetivos estratégicos de las universidades, las cuales deben ser respuestas proactivas a los requisitos del mercado laboral, a los cambios internacionales previstos y al énfasis creciente por elevar los niveles de la calidad universitaria. 


\section{Referencias}

CEPAL. (2000) América Latina y el Caribe en la Transición hacia una Sociedad del Conocimiento.

CHEPS (1999). From the Eye of the Storm, Higher Education's Changing Institution Holanda, University of Twente.

Club Gestión de Calidad. (1999). Método para mejorar la calidad de la educación superior basado en el modelo de la EFQM. Madrid.

Courtright, C. y San Sebastián, C. (1999). Estrategia para la creación de una sociedad de aprendizaje. http://www.conectando.org.sv/Estrategia/index.htm

CSUCA. S.f. Acreditación: Documentos de SICEVAES.

EFQM: 'Introducción a la Excelencia' y 'Conceptos fundamentales de la Excelencia', www.efqm.org.

James, P. (2001) Gestión de la Calidad Total, Un texto introductorio, Madrid. Pearson Educación

Matiru B; A. Mwangi y R. Schlette. (1995). Teach your best, A Handbook for University Lecturers, German Foundation for International Development (DSE).

Nava, V. M. y Jiménez, A. R. ISO 9000:2000, Estrategias para implantar la norma de calidad para la mejora continua. México. Limusa.

Picardo, O. J. Pedagogía informacional: enseñar a aprender en la sociedad del conocimiento. El Salvador. Universidad Francisco Gavidia,

Quesada, M. A.; Cedeño, M. A. y Zamora, J. M. (2001). El diseño curricular en los planes de estudio: aspectos teóricos y guía metodológica. Heredia. Editorial Universidad Nacional.

Sociedad del Conocimiento. www.sociedaddelconocimiento.net

Universidad Nacional. (2002). Significados de Elementos. Taller Una visión compartida de la docencia en la Universidad Nacional. Heredia. 
Universidad Nacional. (2003). Los estudiantes en el futuro escenario de la Universidad Nacional. Memoria. Heredia.

Universidad Nacional. (2004). Plan Global Institucional 2004-2014. Heredia.

Universidad Politécnica Rótterdam. (2003) Positionpaper 2003 - 2007. www.hogeschool-rotterdam.nl.

Zamorano. s.f. Aprender haciendo. http://www.zamorano.edu/estudios/ index.html 